\title{
Embryological and Floral Effects Attributable to Deficiencies Following Crosses with X-Rayed Pollen of Lilium regale W.
}

\author{
Johanna Elizabeth Phay Poole, D. T. Morgan, Jr. and R. D. Rappleye ${ }^{1}$ \\ Department of Botany, University of Maryland, College Park, \\ Maryland, 20742 U.S.A.
}

Received May 4, 1977

Spontaneous multiple seedlings, arising from polyembryonic seeds, occur in a number of different angiosperms. It is well-known that chromosomal variants, particularly in regard to number, are often abundant among multiple seedlings. Previously, striking increases in the frequency of biembryonic seeds of Lilium regale E. H. Wilson were observed following $X$ irradiation of the pollen (Morgan and Rappleye 1951), but the resulting seedlings did not survive. The present study was undertaken to induce polyembryonic seeds of $L$. regale and grow the multiple seedlings produced to maturity in order to detect phenotypic and chromosomal deviations. Classification of the plants from polyembryonic seeds should allow conclusions concerning the origin of the extra embryos.

\section{$\mathrm{X}$ irradiation of the pollen}

Flowers from plants grown in the greenhouse were emasculated prior to anthesis and the anthers placed on paper towels for subsequent dehiscence. Samples of pollen from each flower were stained with aceto-carmine and observed to be wellfilled and normal in appearance. A dosage of $500 \mathrm{r}$ was previously found to be effective in inducing biembryonic seeds of this species (Morgan and Rappleye 1951). Accordingly, the same dosage was applied at the rate of $170 \mathrm{r}$ per minute by a General Electric Maximar 100 X-ray unit. A target distance of $30 \mathrm{~cm}$ was used and the unit operated at $5 \mathrm{ma}$ and $100 \mathrm{kvp}$ with added filtration of one-quarter $\mathrm{mm}$ of aluminum. Sixty-seven mature capsules were obtained following crosses with the irradiated pollen.

\section{Induced polyembryony}

Seeds of lily are quite thin and, when observed with transmitted light, the embryos can be detected with varying degrees of clarity. This method, although not completely accurate, permits the rapid screening of large numbers of seeds, thereby assuring a selected $X_{1}$ population having a high frequency of embryological effects due to the treatment. A total of 27,534 seeds were examined in this fashion.

1 Scientific Article No. A2307, Contribution No. 5308 of the Maryland Agricultural Experiment Station, Department of Botany, College Park, Maryland, 20742, U.S.A. Based in part on a thesis presented by Johanna Elizabeth Phay Poole to the Faculty of the Graduate School of the University of Maryland in partial fulfillment of the requirements for the Degree of Master of Science. 


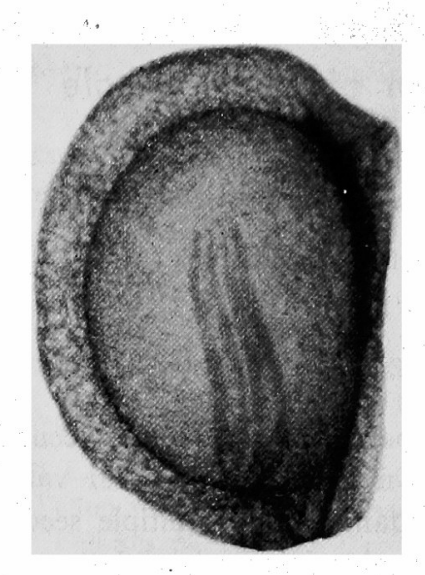

Fig. 1. X-ray induced biembryonic seed of L. regale.
Monoembryonic seeds with embryos of varying sizes comprised 15,387 of the total $\mathrm{X}_{1}$ population; 12,031 of the seeds lacked embryos, 58 were classified as polyembryonic (Fig. 1) and 58 as possibly polyembryonic. In contrast, only one spontaneous biembryonic seed was observed among 13,610 seeds of $L$. regale from crosses with untreated pollen (Morgan and Rappleye 1951).

Prior to germination of the polyembryonic and possibly polyembryonic seeds, 60 apparently normal $X_{1}$ seeds were planted shallowly in quartz sand and placed in a mist chamber in the greenhouse. One twin seedling was detected in the resulting population. Following the satisfactory germination of these seeds, the polyembryonic and possibly polyembryonic seeds were similarly planted. Light

Table 1. $\quad \mathrm{X}_{1}$ multiple seedlings of $L$. regale from seeds classified in regard to numbers of embryos*

\begin{tabular}{lccc} 
Seed class & Number of seeds & $\begin{array}{c}\text { Number of seeds producing twins or triplets } \\
\text { Twins }\end{array}$ & $\begin{array}{c}\text { Triplets } \\
\text { Polyembryonic }\end{array}$ \\
Possibly polyembryonic & 58 & 35 & 2 \\
Apparently normal & 58 & 3 & 1 \\
\hline
\end{tabular}

* Seeds unaccounted for as multiple seedlings either failed to germinate or produced individual seedlings.

applications were occasionally made of soluble fertilizer (20-20-20 analysis with trace elements). Table 1 presents the classification of the $\mathrm{X}_{1}$ multiple seedlings after germination. Multiple seedlings that survived the seedling stage were potted in soil prior to the collection of root tips for chromosomal determinations. Mortality continued after transfer to pots. Twenty-three sets of twins, seven surviving members of twins, and two survivors from each of two sets of triplets grew following transplanting. Accordingly, $57 \mathrm{X}_{1}$ plants from multiple seedlings were obtained.

\section{Mitotic analysis}

Root tips were collected without pretreatment and fixed in three parts of 95 percent ethanol and one part of glacial acetic acid. After storage at $-15^{\circ} \mathrm{C}$, the root tips were softened in equal parts of concentrated hydrochloric acid and 95 percent ethanol for five minutes. Following transfer to 70 percent ethanol for a 
minimum of five minutes, the root tips were smeared in aceto-carmine. Due to unsatisfactory chromosomal distribution, precise analysis could not be accomplished. However, it was established that neither haploid nor polyploid members of the multiple seedlings were present.

In order to facilitate mitotic analysis, additional collections of root tips were
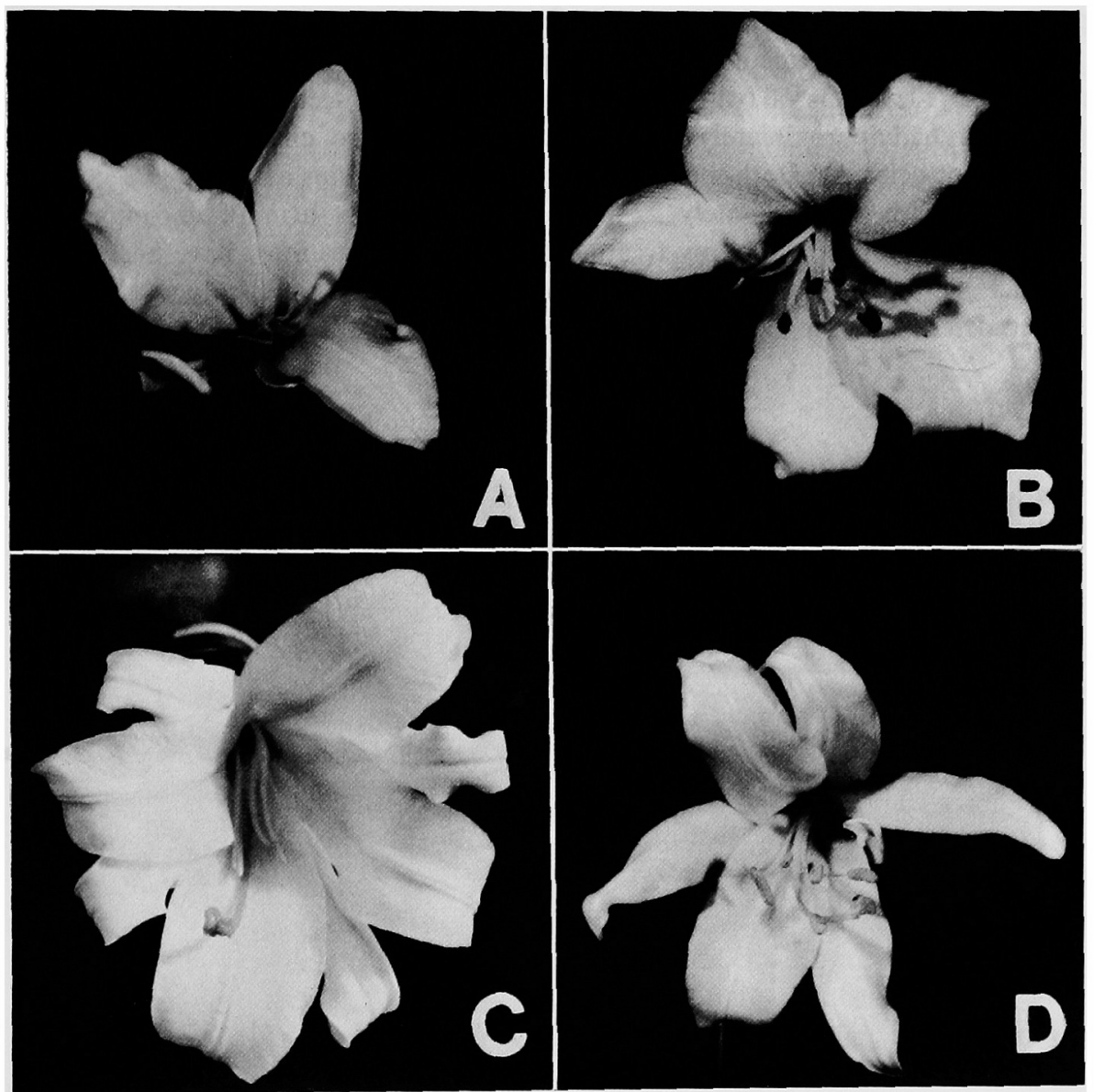

Fig. 2. A-D. Flowers of $X_{1}$ plants from biembryonic seeds. A, two sepals and two petals. One sepal is much reduced. Anthers have been removed to show reduced sepal. Approximately 65 percent of the long arm of chromosome $\mathrm{K}$ is deficient. B, twin of $\mathrm{A}$ and carrying the same deficiency. This flower has three sepals, two petals and five stamens. Two filaments with no anthers are also present. C, four sepals and four petals. Anthers have been emasculated. D, the tip of an extra sepal is visible at right center. Perianth parts are somewhat elongated and loosely arranged. Deficient for approximately 22 percent of the short arm of chromosome B.

made. These collections were pretreated following the method of Stewart (1947) by immersion in 0.2 percent colchicine for 30 minutes followed by 90 minutes rinse in water. The same procedure that had been followed for the untreated root tips was then employed. Analyses of the chromosomal complements were made 
by photographing characteristic cells and projecting the images on paper by means of a photographic enlarger. The outline and centromere position of each chromosome were traced on paper. The drawings were cut out and matched into pairs according to the classification of Stewart (1947). Differences or similarities between homologues were determined by the following method. A compass was used as a divider. Successive straight portions were measured and marked on paper until the whole of a chromosome was drawn linearly and measured.

It was possible to analyze 55 of the $57 \mathrm{X}_{1}$ plants following the preceding procedure. Two sets of twins were heterozygous for detectable deficiencies. One set of twins had approximately 65 percent of the long arm of chromosome $\mathrm{K}$ from the male parent deleted. Both members of another set of twins also shared a common deficiency, with approximately 22 percent of the short arm of chromosome B deleted as a result of the X-ray treatment. Smaller losses must have been present in other $\mathrm{X}_{1}$ plants, but the complements appeared normal since only gross changes can be detected in the chromosomes from root tips of this species.

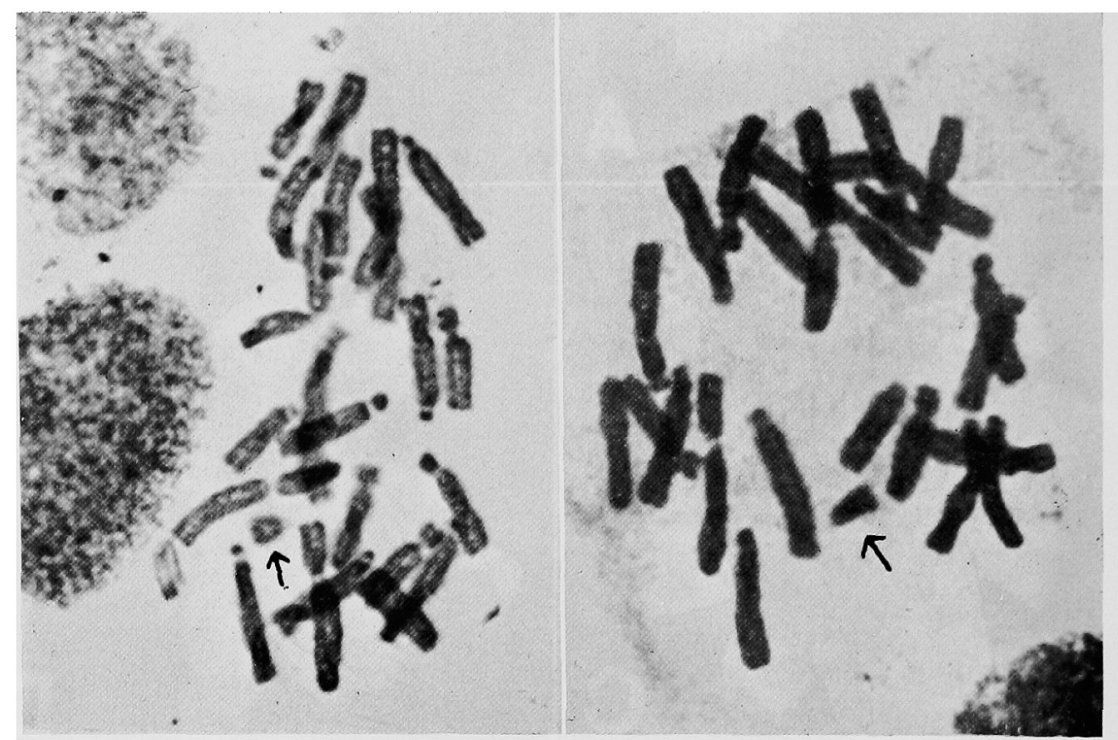

Fig. 3. Same deficiency shared by both members of a set of $X_{1}$ twins. Photomicrographs are metaphase from root tips of different members of the twins. $\times 1520$ on left and $\times 1360$ on right. Measurements of these and other cells disclosed that the loss involved approximately 65 percent of the long arm of chromosome $\mathrm{K}$. This deficiency resulted in twin embryos in the seed and atypical flowers produced by both mature plants.

\section{Discussion and conclusions}

Cooper (1943) found haploid-diploid twin embryos in post-fertilization studies of seven species of Lilium. In approximately one percent of the ovules, a synergid was stimulated to divide, resulting in twin haploid-diploid embryos. However, the haploid usually disintegrated at an early developmental stage, being unable to complete with the more vigorous accompanying diploid embryo. Presumably, 
competition between the two embryos could be decreased by the presence of deleterious X-ray induced deficiencies in the sexual embryo and thus permit survival of some haploids in the present study.

The haploid member of a set of spontaneous twin seedlings is generally smaller than the associated diploid (Randall and Rick 1945, Morgan and Rappleye 1954). Differences in size between the two embryos within a seed and in the resulting two seedlings were also often apparent in the X-ray induced twins of $L$. regale. The first known haploid in angiosperms was obtained in Datura stramonium following X-ray treatment of the pollen (Blakeslee et al. 1922) and the subsequent rather numerous reports of X-ray induction of haploids have been summarized by Magoon and Khanna (1963). Since haploids occur among twin seedlings and have been detected following X-ray treatment of the pollen, it seemed plausible that haploids would be present among the members of the $X$-ray induced twins. However, none occurred, and a different explanation must be sought for the occurrence of the $\mathrm{X}$-ray induced extra embryos.

The apomictic or sexual origin of polyembryonic seeds can be determined by direct observation of development of embryos or by the genetic classification of multiple seedlings from marked crosses. Neither method is applicable for determination of the origin of polyembryonic seeds of $L$. regale due to the low frequency of seeds with more than one embryo and the lack of known marker genes in this species. Nevertheless it is possible to demonstrate that cleavage polyembryony is concerned in the X-ray induction of twin embryos. Two sets of twins were partially conjoined and may be accounted for by cleavage occurring relatively late in ontogeny. It seems apparent that cleavage could occur in earlier phases of development, resulting in unattached twins. Chromosomal analysis and observations of fioral morphology substantiate this assumption.

Floral modifications were abundant among the members of the $X_{1}$ twins (Fig. 2). Notched and ruffled petals and sepals occurred, resulting in distinctive and occasionally attractive variants. Normal flowers of regal lily have three sepals, three petals, and six stamens. Increases and decreases in the number of stamens and perianth parts were frequent. The two members of a set of twins were similarly atypical. Figs. $2 \mathrm{~A}$ and $\mathrm{B}$ show reduction in numbers of parts in the flowers of two members of a twin. Both members had in common a deficiency for approximately 65 percent of the long arm of chromosome K (Fig. 3). Since the two unattached members had similar phenotypes and were identically deficient, it is concluded that they arose by cleavage in early phases of development. It is also concluded that deficiencies were primarily responsible for the variants in floral morphology. Fig. 2D shows a flower with open form and long, narrow perianth parts. An extra sepal is present. This plant and its twin were identically deficient for approximately 22 percent of the short arm of the B chromosome contributed by the sperm from the X-rayed pollen. In addition to the gross losses, smaller deficiencies also must have occurred and were similarly responsible for development of extra embryos and floral modifications. 


\section{Summary}

Polyembryonic seeds of Lilium regale $\mathrm{E}$. $\mathrm{H}$. Wilson were induced by $\mathrm{X}$ irradiation of the pollen with $500 \mathrm{r}$. Mitotic analyses of 55 plants arising from the polyembryonic seeds disclosed that two sets of identical unattached twins were heterozygous for different gross deficiencies. Highly atypical flowers were produced by the deficient plants. Smaller deficiencies, beyond the limit of detection, undoubtedly were present in other sets of twins. Two sets of conjoined twins occurred. Increases or decreases in the number of floral parts were numerous; both members of a set of twins had similar flowers, indicating a common origin. It is concluded that X-ray induced deficiencies were responsible for cleavage during embryological development and the subsequent formation of atypical flowers.

\section{Literature cited}

Blakeslee, A. F., Belling, J., Farnham, M. E. and Bergner, A. D. 1922. A haploid mutant in the Jimson weed, Datura stramonium. Science 55: 646-647.

Cooper, D. C. 1943. Haploid-diploid twin embryos in Lilium and Nicotiana. Amer. Jour. Bot. 30: 408-413.

Magoon, M. L. and Khanna, K. R. 1963. Haploids. Caryologia 16: 191-235.

Morgan, D. T., Jr. and Rappleye, R. D. 1951. Polyembryony in maize and lily following X irradiation of the pollen. Jour. Hered. 42: 90-93.

- and - 1954. A cytogenetic study on the origin of multiple seedlings of Capsicum frutescens. Amer. Jour. Bot. 41 : 576-585.

Randall, T.E. and Rick, C. M. 1945. A cytogenetic study of polyembryony in Asparagus officinalis L. Amer. Jour. Bot. 32: 560-569.

Stewart, R. N. 1947. The morphology of somatic chromosomes in Lilium. Amer. Jour. Bot. 34: 9-26. 